\title{
The Design of a Shoe for the Analysis of Ambulation Pattern for Diverse Age-Cohort
}

\author{
Mehwish Faiz*, Choudhary Sobhan Shakeel, Munira Muhammadi Zariwala, Umer Hassan, Fatema Ilyas \\ Department of Biomedical Engineering, Ziauddin University, Faculty of Engineering, Science, Technology, and Manage- \\ ment, Karachi, Pakistan. \\ ${ }^{*}$ Corresponding author: mehwish.faiz@zu.edu.pk
}

\section{Abstract}

\begin{abstract}
Ambulation is one of the important activities of daily life aided by musculoskeletal system. However, with ageing due to muscle weakness and decreased range of motion of joints, different walking abnormalities may occur. These abnormalities may result in deviation of the spatio-temporal gait parameters. Mostly, alteration in pattern of locomotion has been observed with the increase in the age of an individual. This variation in the ambulation pattern and subsequent decrease in the gait parameters pertains to the onset of musculoskeletal disorders such as osteoarthritis and osteoporosis as well as various postural abnormalities. Hence, wearable technologies can aid individuals to monitor their gait parameters efficiently and alert them in case there is a significant decrease. This paper describes the design of an Arduino based monitoring system which analyzes the two significant gait parameters: step length and ground reaction force (GRF). A diverse age cohort consisting of 70 healthy individuals with ages in the range of 18 years to 80 years were recruited for this study. The shoe prototype measures the step length and ground reaction force (GRF). This is accomplished by making use of sensors including a Force Sensitive Resistive sensor (FSR) and an Ultrasonic sensor. The FSR sensor exhibits the ground reaction force that the ground exerts on a body that comes in contact with it; whereas, the ultrasonic sensor measures the step length which pertains to the distance between the initial contact of one foot and the point of initial contact of the other foot. The results of the study show that with age there is a significant decrease in the ambulation parameters. Young participants were observed to exhibit higher values of step length and ground reaction force (GRF) as compared to older participants who exhibit significant decrease in the parameter values. The results also show that there is a significant difference between the age of the individuals and the step length and ground reaction force values. The decrease in the gait parameters with increasing age permits this shoe prototype to be used for clinical settings in the future and be able to analyze the onset of any musculoskeletal disorder.
\end{abstract}

Keywords-ambulation, step length, ground reaction force, gait parameters, musculoskeletal disorder, postural abnormalities

\section{Introduction}

$\mathrm{F}$ OR an independent living, ambulation is a very important part of life. Gait velocity and qualitative measurements such as step length, ground reaction force, path deviation, and foot clearance can help to predict future conditions of the old people like fall risks and factors which cause disability in ambulation process or walking. Aging is always associated with the gait velocity, which depends on many factors, but primarily depends on the step length. As per

ISSN: 2523-0379 (Online), ISSN: 1605-8607 (Print)

DOI: https://doi.org/10.52584/QRJ.1901.17

This is an open access article published by Quaid-e-Awam University of Engineering Science $\&$ Technology, Nawabshah, Pakistan under CC BY 4.0 International License. statistical survey, gait velocity is maintained till the seventh decade of adult life and after every decade, gait velocity usually declines $12-16 \%$ of the normal range. Many factors are involved in the shortening of step length and reduction in gait velocity, such as hip extension or flexion, reduced pelvic rotation, and reduced ankle plantar flexion power. The most important and decisive factor in the shortening of the step length is ankle plantar flexion power. In plantar flexion power, the muscle's strength is inversely proportional to the age, as the muscle's strength decreases with increasing age of an individual, the plantar flexion power also reduces, which shortens the step length [1]. Gait disturbance is also directly related with different diseases 
and it can be primarily assessed by monitoring the step length and ground reaction force. One of the diseases with gait disturbance as the fundamental sign is the Parkinson's disease (PD). A study was conducted on $\mathrm{PD}$ patients and they were allowed to walk on a treadmill. After interpretation from recorded values, it was concluded that PD patients had variations in step length compared to the healthy subjects [2]. Another parameter is the ground reaction force (GRF), which is affected by aging. Aged people exhibit low ground reaction force as compared to the adults or younger ones, because there is a significant decrease in muscle strength and bone density which decreases their ability to walk and their balancing ability. This compromises their ability to carry out independent life and routine activities. Young people have greatest muscle strength as their bone building cells are functional and do not experience reduction in GRF [3].

Step distance pertains to the length between the feet to the heel strikes. During ambulation, step length is the distance measured between feet, while stride length is the distance measured between one-foot step and the next foot step of the same foot. Hence, it can be concluded that stride length is double the distance of a step. Therefore, if the stride length of a healthy male is 5 feet or 60 inches approximately, and a healthy woman is about 4.4 feet or 53 inches, then step length would be calculated as the half of these values. The importance of step length lies in the fact that normal walking velocity in a healthy individual is the one that takes the least energy consumption per unit distance. Ground reaction force is the force that ground will exert on a body that comes in contact with it. Ground reaction force is particularly important in analyzing falls and other conditions when carrying out a comparative analysis between young and older individuals [4].

\section{Literature Survey}

Age is a factor which results in decreased step length that causes a decreased velocity of gait cycle. Several studies depict the relationship between step length and kinetics of joints. Twenty six older individuals with an average age of 79 and 32 young individuals with an average age of 26 were selected from the community and utilized for a study [1]. During a normal gait cycle, three-dimensional gait kinematic and kinetic parameters were observed. The older age group individuals had a $10 \%$ lesser step length than the younger as per the normal and general conditions. Decreased ankle plantar flexion during second stance phase reduces the ankle plantar flexor power in older individuals.
Ankle power was related to plantar flexor power during the late stance. Older individuals were not able to elevate their ankle plantar flexor power; however, an elevation in their hip flexor power was observed. This study was useful for maintaining step length in older individuals, but other spatial and temporal parameters along with step length could have been taken into account as well. Deviation in the step size and the duration of the double support is a key indication to distinguish between old people who fall easily than old people who do not fall easily and young individuals. To observe the spatial and temporal characteristics of gait cycle, their ambulation pattern for at least three strides was observed [5]. The results showed that old individuals that do fall easily exhibit a much smaller step length and elongated double support duration. The step length deviations observed for old individuals that could fall easily were twice than that exhibited by old individuals who did not fall easily. These deviations in step length may be a vital parameter for analyzing postural abnormalities.

Injuries resulting from fall are one of the most common problem faced by elderly. With the help of recent studies, focus has been given on the variations that occur in step kinematics including step length and its association to falling. The variations of the step kinematics showed a linear relation with the cadence (number of steps). A treadmill with the proper set up was utilized to analyze all spatial and temporal parameters. This study was carried out to determine the impact of age, speed of walking, and use of handrail on the variations in step parameters. Eighteen young individuals of average age of 27 years and twelve healthy adults of average age of 73 years were engaged in the study. Three parameters were analyzed for variations including step time, step width, and step length. The results showed that the deviations in step width of elderly were noticeably higher than those of young individuals. Variations in step width and step length were only governed by the use of hand rail. The current results and those of previous studies exhibit steady relationship between age and step width variations. Since deviation in step parameters is related to falls, further research needs to be carried out [6].

A study [7] investigated the correlation between the knee angular velocity and the ground reaction force of the young and older individuals as the person ages. Pearson's correlation test was performed to find out the correlation between the two groups. The significance value found was $p=0.000$ which means that there was a direct correlation between velocity and the force exerted. Young group had greater mean force magnitude (1.39) as compared to the elderly (1.15). 
The study concluded that when age increases, there exists a positive correlation with the declination of the muscle force.

In case when an underlying disease cannot be found, the term 'senile gait disorder' is used to emphasize gait problems in older adults [8]. A study compared group of healthy individuals in their twenties and group of healthy individuals in their seventies and its results exhibited a 10\%-20\% decrease most notably in the gait velocity and stride length of the older population [9]. A comparative analysis of gait parameters between healthy young women and healthy elderly women was conducted for which T-test was utilized. The results of the study exhibited that the group of healthy elderly women had smaller values of step length, stride length, range of motion of the ankle, and gait velocity when compared to the group of healthy young women [10].

Different techniques and methodologies have been used for the analysis of gait parameters including step length and GFR. In [11], step length is calculated by making individuals walk on treadmill and recording their activity by heel markers. The calculated step length is then used to find out the value of stride length by software which increases the efficiency of the system and saves the time. However, the calculated value of stride length has minor difference and inconsistent from the expected value because summation of right and left step length to yield stride length is more appropriate for the analysis of gait parameters while walking on ground rather than on treadmill.

Another method for the measurement of step length is to make individuals walk on a pressure sensitive paper for the recording of gait parameters [12]. However, this method is not very reliable. Although this method is simple and does not need much hardware, there are more improved methods for the calculation of step length. A 3-dimensional voxel system built with a webcam is also utilized for examination of gait cycle and its parameters at home for older individuals [13]. The 3-dimensional voxel was constructed to gather information regarding walking velocity, step length, and step time. The gait characteristics were extracted via GAIT rite mat and VICON motion capture system as software. The study showed that the individuals who were tested with GAIT rite exhibited different gait characteristics than the individuals who were tested in an unstructured scenario. Two limitations of this study were frame rate and voxel resolution.

In [15], a wearable computer device was used for the measurement of step length by placing the sensors on the waist of an individual by taking into account the movement of trunk as a key factor. This device comprises of cameras, microprocessor, and other components to acquire motion data. The data acquired is further processed by various signal processing stages and then vertical component of acceleration is used to find out the value of step length. However, there are chances of error in the value of step length as the signal has to pass through multiple stages of filtration and pre-processing. Moreover, step length can be estimated with improved results, if we are taking the readings from the limbs of an individual [14]. For the measurement of GRF, a sensor system is used, which makes use of a shoe. The force sensors collect the signals which are then sent to a microcomputer for further processing.

Measuring gait velocity with step length is vital as gait velocity has been observed to be linked with predicting heart failure in adults [16] and chronic obtrusive pulmonary disease [17]. In [18], knee and hip kinetics and kinematics of the subjects with different step lengths and ground reaction force were evaluated pertaining to joint movements of knee and hip. The results showed that both factors are largely associated with step length and tend to vary with age. Ambulation pattern of different aged group individuals are significant tools for assessing and relating different diseases with disturbed ambulation pattern. These parameters are of great importance in not only understanding and comparing kinetics and kinematics, but can also be utilized for executing a better analysis of any postural abnormality [5]. In another study [19], shoes for analyzing gait were designed involving healthy and post stroke hemiplegic patients. FSR and camera technology was utilized and the results of the study showed $1.24 \%$ absolute error for spatial parameters and $1.12 \%$ absolute error for temporal parameters. In [20], a comparative analysis pertaining to walking bare foot and with minimum footwear was elaborated between young and older adults. The results of the study showed that while adhering to wearing footwear for ambulation, gait parameters tend to exhibit less variations as compared to when walking bare foot.

Compared to the work presented in [19], our proposed method stands apart by recruiting a diverse age cohort for our study which include individuals with age in the range of 18 years and 80 years whereas only 24 participants were recruited for the study in [19]. Furthermore, our proposed shoe prototype measures a significant spatial parameter in the form of step length as well as ground reaction force whereas the work proposed in [19] only measures spatio-temporal parameters. An external camera is required for the measurement of spatio-temporal parameters in [19] which 
tends to increase cost and computational complexity. Our shoe prototype enables real-time step length measurement accurately with an ultrasonic sensor hence; it is more cost-effective and requires less time for computation of the parameter values. The total cost of the shoes designed in [19] were $\$ 90$; whereas, our designed prototype costed $\$ 10$.

Compared to the work in [20], we recruited a more diverse age cohort for ambulation pattern analysis with the measurement of vital gait parameters that tend to indicate about any musculoskeletal system disorder or postural abnormality. Furthermore, the mentioned techniques in the literature survey require expensive hardware and software to track the ambulation pattern. Moreover, these devices are either measuring step length or GRF separately. Therefore, we propose the design of a shoe prototype which is compact, economical, portable, and can be used anywhere to examine an individual's gait pattern by measuring step length and GRF. This wearable technology in the form of a shoe prototype particularly enables older individuals in the age group of 70 years and 80 years to keep a track of their step length and GRF values continuously, and in case of any considerable nonconformity, they can seek medical assistance in due time. This will help such individuals to adhere to a robust diagnosis and treatment regimen in case of any musculoskeletal or postural abnormality.

\section{Methodology}

A shoe prototype is designed for the analysis of gait parameters including step length and ground reaction force. It consists of a wearable shoe integrated with two sensors, as shown in Figure 1. The electrical connections were adequately insulated. The connections of the FSR were properly embedded within the sole of the shoe, so that external forces do not produce undesirable results when analyzing an individual's ambulation pattern. The first sensor used in the prototype is Force Sensitive Resistive sensor (FSR), and the second one is an ultrasonic sensor. Figure 2 shows the block diagram of the shoe prototype with its components. Figure 3 illustrates the flow process that pertains to working of the shoe prototype. FSR is used to calculate the force exerted by the subject on the ground, and the ultrasonic sensor is used to calculate the step length in centimeters. The sensors were placed on the heel side of the shoe and calculated the load distribution from the heel.

FSR is fabricated with two layers of substrate. Furthermore, a conductive film and plastic spacer are also incorporated. When an individual applies force on the

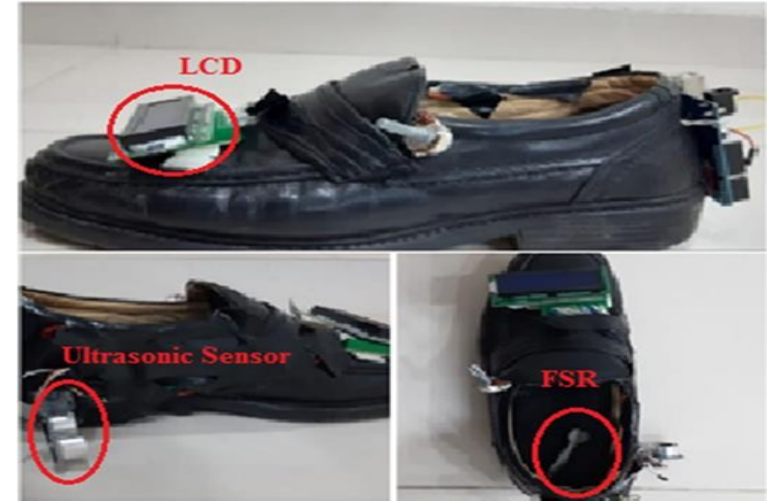

Fig. 1: Shoe prototype with its components

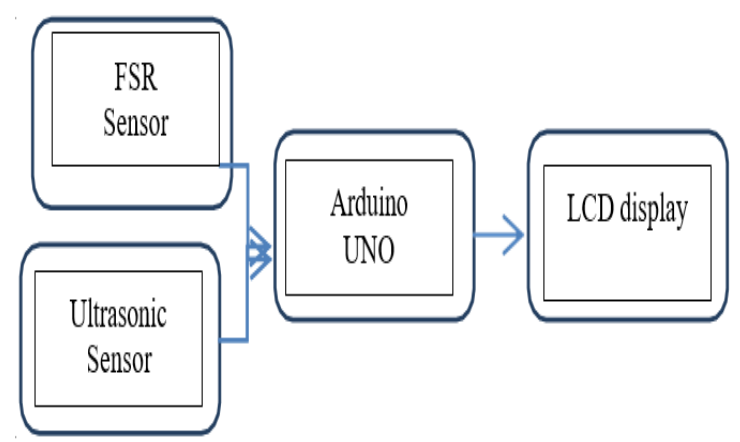

Fig. 2: Block diagram of the proposed shoe prototype

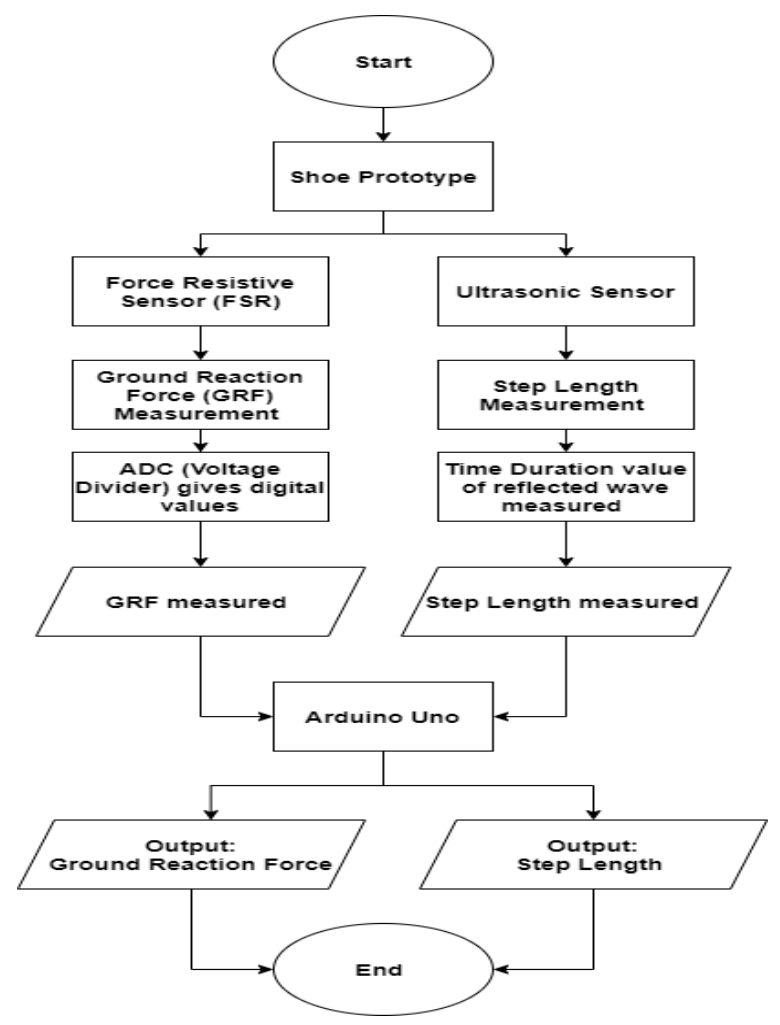

Fig. 3: Flow Process of the proposed shoe prototype 
FSR, it calculates the pressure applied as force per unit area. The force reading is displayed on the LCD. Whenever the subject applied force on the sensor, its resistance and conductance changed due to which force was calculated. When an individual applies force on the FSR, the conductive film changes its shape due to which the spacer opening allows the air to pass through and the film makes contact with the substrate layers. Hence, with increasing pressure, the contact between film and the substrate layers increases which decreases the resistance and in turn a force is measured. Equation 1 shows the relationship by which the FSR computes the force value. Initially when no force is applied on the FSR, the conductance represented by $\rho$ is, 0 and the resistance represented by $R$ is infinite. When an individual applies pressure on the FSR, the conductance tends to increase, whereas, the resistance decreases to 0 . Hence, it can be concluded that the FSR demonstrates an indirect relationship between conductance and resistance and this is shown by Equation 2.

$$
\begin{gathered}
F=P / A \\
\rho \propto 1 / R \\
G R F=W=m g \\
G R F=\sqrt{(G R F x)^{2}+(G R F y)^{2}} \\
G R F x=G R F \cos \theta \\
\sin \theta=\operatorname{perp} / h y p=G R F y / G R F \\
G R F y=G R F \sin \theta
\end{gathered}
$$

Figure 4 represents the schematic design for the proposed shoe prototype to calculate ground reaction force using an FSR. The circuit was simulated on the FRITZING software, which can be easily accessed from the open source. This software has been utilized due to the availability of the library of the desired component. Figure 4 demonstrates the connections of the FSR with Arduino Uno. The following steps have been executed for the connections.

- One of the pins of the FSR is connected to the $5 \mathrm{~V}$ pin of Arduino Uno

- The other pin of the FSR is connected to the $A_{0}$ pin of the Arduino Uno

- A $10 \mathrm{k} \Omega$ resistor is connected between the $A_{0}$ and ground connection

Figure 5 demonstrates the connections of the Ultrasonic sensor with Arduino Uno. The following steps have been executed for the connections.

- The trigger pin of the ultrasonic sensor is connected with pin 9 of Arduino Uno

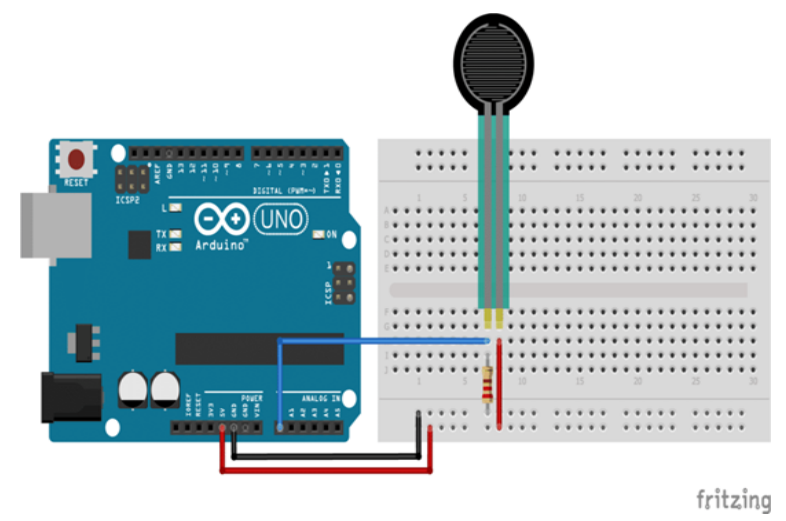

Fig. 4: Circuit diagram of the proposed prototype with FSR and Arduino Uno

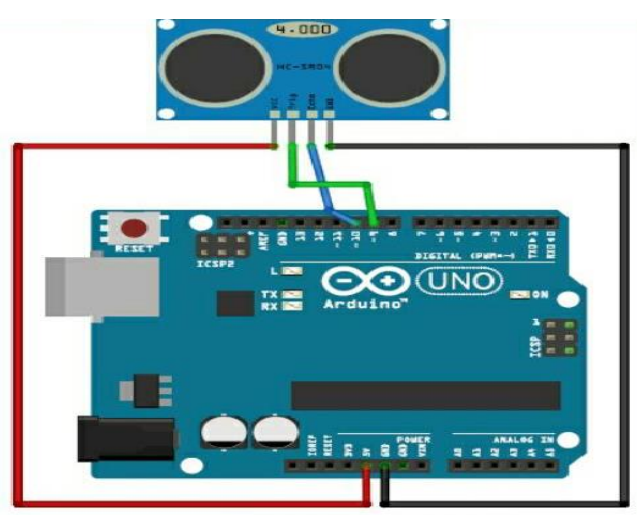

Fig. 5: Circuit diagram of the proposed prototype with ultrasonic sensor and Arduino Uno

- The echo pin of the ultrasonic sensor is connected with pin 10 of Arduino Uno

- The ground and VCC pins of the sensor have been connected with the ground and VCC of the Arduino Uno.

In order to measure the force from the FSR, there is a need for a voltage divider circuit. Since the value generated by FSR in analog in nature, it cannot be read by the microcontroller directly. Hence, a voltage divider circuit will serve as analog to digital converter (ADC) so that the force value can be read and computed in the form of voltages. Equation 8 gives the voltage divider rule,

$$
V_{\text {out }}=V_{\text {in }} \times F R /(R+F R)
$$

Where $V_{\text {out }}$ and $V_{\text {in }}$ are the output and input voltages, respectively, $F R$ is the force resistance, and $R$ is the voltage divider resistance.

Table 1 shows the data collected via the random force measurement from the shoe prototype. The data was acquired from a healthy participant in the age group of 20 years to 30 years. The weight of the individual 


\begin{tabular}{|l|l|l|}
\hline $\begin{array}{l}\text { Force } \\
\text { (gm) }\end{array}$ & $\begin{array}{l}\text { Resistance } \\
\text { (ohms) }\end{array}$ & $\begin{array}{l}\text { Voltage } \\
\text { (volts) }\end{array}$ \\
\hline 0.47 & 3301060 & 3.7 \\
\hline 10.9 & 146965 & 2.7 \\
\hline 2.37 & 657628 & 3.2 \\
\hline 173 & 8962 & 1.05 \\
\hline 864.5 & 2160.36 & 0.86 \\
\hline 1188 & 1308 & 0.2 \\
\hline
\end{tabular}

TABLE 1: Random force measurement from the prototype

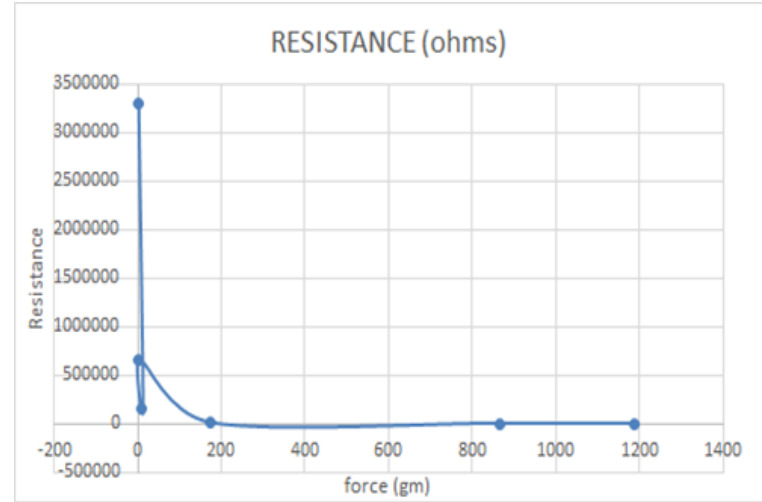

Fig. 6: Graphical representation of random force measurement from the proposed prototype

was $67.9 \mathrm{~kg}$ and the height was $167.94 \mathrm{~cm}$. Hence, the computed body mass index (BMI) was 24.1 which is in the normal weight range. Figure 6 demonstrates the relationship between the force, resistance and voltages. It can be seen that as force increases, both parameters decrease linearly. Step distance pertains to the length between the feet to the heel strikes. During ambulation, step length is the distance measured between feet, while stride length is the distance measured between one-foot step and the next foot step of the same foot. Hence, it can be concluded that stride length is double the distance of a step and then step length would be calculated as the half of these values. The relationship between step length and stride length is shown by Equation 9 and Equation 10. SrL and StL pertain to stride length and step length, respectively.

$$
\begin{gathered}
S r L=2 S t L \\
S t L=1 / 2 S r L
\end{gathered}
$$

To calculate the step length, an ultrasonic sensor has been used. In an ultrasonic sensor, the waves have a speed of $330 \mathrm{~m} / \mathrm{s}$ at room conditions. The sensor calculates the time taken by the ultrasonic wave to reflect back and get received by the echo pin which was specified a high logic. The measurements were initiated, by making the trigger pin high, at $10 \mu \mathrm{s}$, and then it was turned off. The pulse was sent at the frequency of $40 \mathrm{~Hz}$. After the wave struck an object and was reflected back, the echo pin was given a high logic for the same amount of time. The duration of the time for which the echo pin stayed high was calculated by means of the microcontroller. The sensors are horizontally attached at the back of one foot. Therefore, when the subject took a step, the duration of the reflected wave from one heel to another heel was calculated. This time duration aids in the computation of the step length in the form of distance with respect to time and is represented by Equation 11.

$$
d=\frac{s}{t}
$$

To implementation of the shoe prototype has been executed via accumulation of readings from a diverse cohort consisting of 70 individuals having ages in the range of 18 years and 80 years. For this purpose, an outlined area of 12 sq.ft was selected. Then, participants wearing the prototype were allowed to walk in order to record their step length and ground reaction force. Readings were taken from different age group of healthy people without any prior history of postural and musculoskeletal injury or low back pain.

Table 2 contains the information pertaining to the acquired readings from the individuals with the aid of the designed prototype including their demographic data to analyze the significance of variation between ambulation pattern and age. Statistical analyses were performed using SPSS Statistics 22.0 (IBM SPSS Inc., Chicago, USA). For the gait parameters, two-way repeated measures ANOVA was executed in order to evaluate the effect of participant age on step length and ground reaction force (GRF). A $p<0.001$ was required for differences to be considered statistically significant.

\section{Results}

The acquired measurements in Table 2 show that young individuals exhibit higher values of step length, but as the individual ages, the value of step length decreases. It is also evident from Table 2 that older individuals have reduced ground reaction force as compared to young people. Moreover, two individuals with same age having different weight and height possess different values of step length and ground reaction force with the conclusion that the individual having increased height and weight have higher value of step length and ground reaction force as compared to other individual of the same age. Figure 7 shows the relationship of age and step length with the conclusion that as the age of an individual increases, the step 


\begin{tabular}{|c|c|c|c|c|c|c|c|c|c|c|c|}
\hline $\begin{array}{l}\text { Partici- } \\
\text { pants }\end{array}$ & Age & $\begin{array}{l}\text { Height } \\
(\mathrm{cm})\end{array}$ & $\begin{array}{l}\text { Weight } \\
\text { (g) }\end{array}$ & $\begin{array}{l}\text { Step } \\
\text { Length } \\
\text { (cm) }\end{array}$ & $\operatorname{GRF}(\mathbf{N})$ & $\begin{array}{l}\text { Partici- } \\
\text { pants }\end{array}$ & Age & $\begin{array}{l}\text { Height } \\
(\mathrm{cm})\end{array}$ & $\begin{array}{l}\text { Weight } \\
\text { (g) }\end{array}$ & $\begin{array}{l}\text { Step } \\
\text { Length } \\
(\mathrm{cm})\end{array}$ & $\begin{array}{l}\text { GRF } \\
(\mathrm{N})\end{array}$ \\
\hline 1 & 18 & 175.26 & 54000 & 100 & 19.8 & 36 & 64 & 189 & 52000 & 64 & 9.7 \\
\hline 2 & 19 & 167.64 & 50000 & 92 & 19.6 & 37 & 64 & 149 & 67000 & 64 & 10.4 \\
\hline 3 & 20 & 152.4 & 42000 & 96 & 15.8 & 38 & 65 & 175 & 59000 & 60 & 11.6 \\
\hline 4 & 21 & 144.78 & 45000 & 88 & 14.8 & 39 & 65 & 144 & 59000 & 68 & 11.3 \\
\hline 5 & 22 & 154.94 & 38000 & 96 & 12.9 & 40 & 65 & 157.48 & 50000 & 72 & 14.7 \\
\hline 6 & 50 & 164 & 61000 & 64 & 17.7 & 41 & 65 & 152.4 & 60000 & 72 & 16.8 \\
\hline 7 & 50 & 148 & 47000 & 62 & 14.1 & 42 & 67 & 159 & 61000 & 68 & 13 \\
\hline 8 & 50 & 159 & 52500 & 63 & 13.4 & 43 & 67 & 140 & 59000 & 64 & 12.7 \\
\hline 9 & 51 & 149 & 52000 & 62.8 & 14.5 & 44 & 68 & 130 & 62500 & 74.9 & 9.8 \\
\hline 10 & 52 & 152 & 58500 & 60 & 18.3 & 45 & 68 & 175 & 52000 & 74.9 & 12.7 \\
\hline 11 & 52 & 159 & 65000 & 60 & 12.6 & 46 & 69 & 135 & 68000 & 73.5 & 10.4 \\
\hline 12 & 53 & 144 & 55000 & 60 & 11.8 & 47 & 69 & 162 & 61000 & 72.8 & 9.5 \\
\hline 13 & 54 & 162 & 53000 & 60 & 16.6 & 48 & 69 & 165 & 68000 & 71.4 & 13.7 \\
\hline 14 & 55 & 175 & 55000 & 60 & 15.9 & 49 & 70 & 158 & 59000 & 71.4 & 10.9 \\
\hline 15 & 55 & 163 & 53000 & 80 & 17.7 & 50 & 70 & 159 & 56000 & 70.7 & 11.3 \\
\hline 16 & 55 & 162 & 51000 & 72 & 15.1 & 51 & 71 & 165 & 49000 & 70 & 12.3 \\
\hline 17 & 55 & 144 & 52000 & 68 & 13.4 & 52 & 71 & 148 & 52500 & 70 & 9.7 \\
\hline 18 & 56 & 148 & 58000 & 64 & 15.3 & 53 & 72 & 137 & 58000 & 70 & 9.4 \\
\hline 19 & 57 & 157 & 51000 & 72 & 12.7 & 54 & 72 & 138 & 49000 & 67.9 & 10.4 \\
\hline 20 & 58 & 167 & 59000 & 76 & 19.4 & 55 & 73 & 148 & 57000 & 66.5 & 9.8 \\
\hline 21 & 58 & 165 & 55000 & 84 & 16.2 & 56 & 74 & 140 & 52000 & 63.7 & 14.8 \\
\hline 22 & 58 & 149 & 62500 & 72 & 16.1 & 57 & 75 & 159 & 53000 & 63 & 12.7 \\
\hline 23 & 58 & 162 & 49000 & 68 & 12.7 & 58 & 75 & 147 & 61000 & 63 & 14.7 \\
\hline 24 & 59 & 144 & 49000 & 76 & 10.4 & 59 & 75 & 144.78 & 50000 & 63 & 9.8 \\
\hline 25 & 59 & 189 & 59000 & 68 & 12.7 & 60 & 75 & 162.56 & 53000 & 63 & 11.7 \\
\hline 26 & 59 & 167.64 & 59000 & 64 & 19.4 & 61 & 76 & 175 & 46000 & 63 & 12.4 \\
\hline 27 & 60 & 149 & 69000 & 76 & 11.8 & 62 & 76 & 149 & 47000 & 63 & 11.3 \\
\hline 28 & 60 & 139 & 59000 & 80 & 13.4 & 63 & 77 & 152 & 55000 & 62.3 & 13.4 \\
\hline 29 & 60 & 157 & 49000 & 60 & 13 & 64 & 77 & 135 & 51900 & 60.2 & 11.3 \\
\hline 30 & 61 & 158 & 65000 & 72 & 13 & 65 & 78 & 144 & 48000 & 59.5 & 9.3 \\
\hline 31 & 62 & 130 & 58000 & 76 & 11.9 & 66 & 78 & 157 & 61000 & 59.5 & 9.8 \\
\hline 32 & 62 & 152 & 62300 & 76 & 15.9 & 67 & 78 & 148 & 65000 & 56 & 10.7 \\
\hline 33 & 62 & 148 & 47500 & 68 & 10.4 & 68 & 79 & 152 & 47000 & 56 & 12.7 \\
\hline 34 & 63 & 162 & 49000 & 76 & 12.3 & 69 & 79 & 144 & 58000 & 56 & 10.4 \\
\hline 35 & 64 & 160 & 56000 & 68 & 9.6 & 70 & 80 & 165 & 55000 & 55.3 & 12.1 \\
\hline
\end{tabular}

TABLE 2: Demographic data taken with the designed prototype

length decreases. It is depicted in Figure 8 that ground reaction force decreases as the individual age.

The results of the statistical test show that there is a significant difference between the step length and ground reaction force (GRF). Young individuals were observed to exhibit higher values of step length and ground reaction force (GRF) as compared to older individuals. A $p<0.001$ indicates that results are statistically significant. The results are demonstrated by the following expression;

$$
F(1.69)=4.29, \text { where } p<0.001
$$

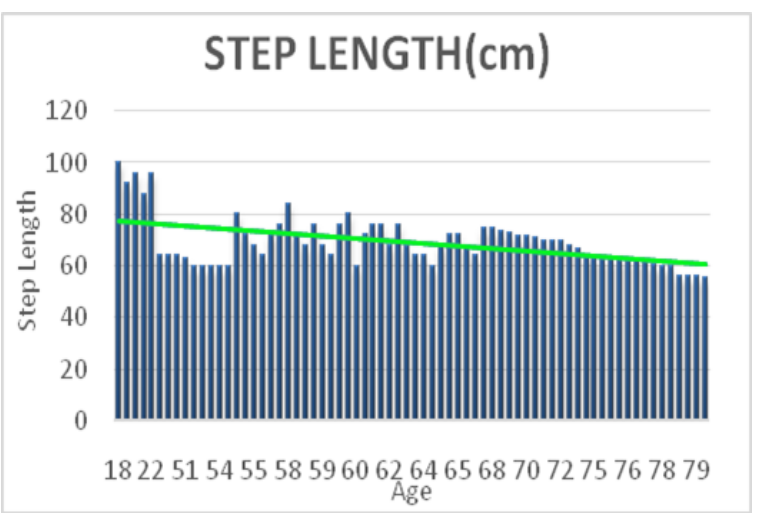

Fig. 7: Graphical representation of step length with increasing age

measure prominent parameters of ambulation pattern: step length and ground reaction force (GRF). These
The proposed shoe prototype pertains to a simple implementation in terms of circuitry and cost to 


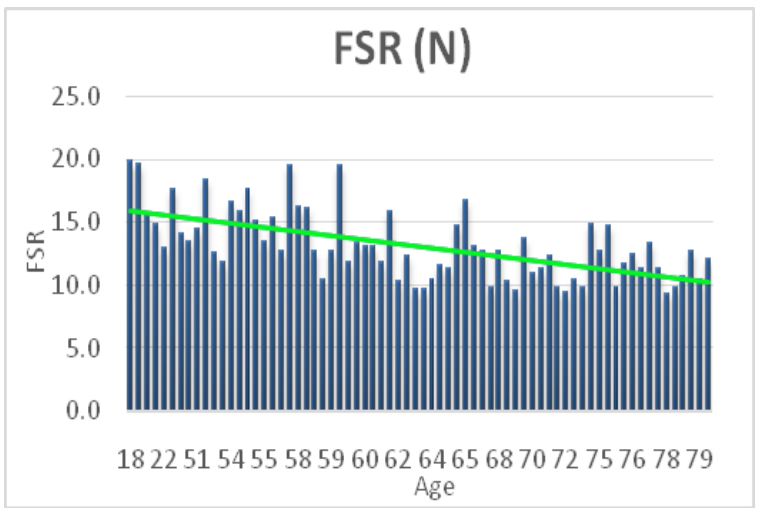

Fig. 8: Graphical representation of ground reaction force with increasing age

parameters are of great importance in not only understanding and comparing kinetics and kinematics, but can also be utilized for executing a better analysis of any postural abnormality. Although our results were promising, one limitation of our study pertains to the number of participants. Hence, in future we aim to incorporate a more diverse age cohort. Moreover, we plan to measure more gait parameters such as cadence and toe out angle and by utilizing different walking patterns. Our shoe prototype has shown to be a reliable, cost effective and a portable solution for analyzing the ambulation pattern in the form of step length and GRF. We also plan to make ergonomic modifications in the shoe prototype, so that it can be used in clinical settings in the future.

\section{References}

[1] J. O. JudgeRoy, B. Davis III, and S. Õunpuu, "Step length reduc-tions in advanced age: the role of ankle and hip kinetics," The Journals of Gerontology Series A: Biological Sciences and Medical Sciences, vol. 51, no. 6, pp. M303M312, 1996.

[2] M. K. Mak, "Reduced step length, not step length variability is central to gait hypokinesia in people with Parkinson's disease," Clinical neurology and neurosurgery, vol. 115, no. 5, pp. 587- 590, 2013.

[3] H. Toda, A. Nagano, and Z. Luo, "Age and gender differences in the control of vertical ground reaction force by the hip, knee and ankle joints," Journal of physical therapy science, vol. 27, no. 6, pp. 1833-1838, 2015.

[4] T. V. Barreira, D. Rowe, and M. Kang, "Parameters of walking and jogging in young adults," International Journal of Exercise Science, vol. 3, no. 1, pp. 4-13, 2010.

[5] G. A. Mbourou, Y. Lajoie, and N. Teasdale, "Step length variability at gait initiation in elderly fallers and non-fallers, and young adults," Gerontology, vol. 49, no. 1, pp. 21-26, 2003.

[6] T. M. Owings and M. D. Grabiner, "Variability of step kinematics in young and older adults," Gait \& posture, vol. 20, no. 1, pp. 26-29, 2004.
[7] D. d. O. Silva, D. C. Thomé, A. S. Ferreira, and F. A. Aragão, "Correlation between vertical ground reaction force and knee angular velocity of young and elderly individuals during stair descent," Re-vista Brasileira de Geriatria e Gerontologia, vol. 18, no. 3, pp. 567-576, 2015.

[8] B. Salzman, "Gait and balance disorders in older adults," American family physician, vol. 82, no. 1, pp. 61-68, 2010.

[9] R. Elble, S. S. Thomas, C. Higgins, and J. Colliver, "Stridedepend-ent changes in gait of older people," Journal of neurology, vol. 238, no. 1, pp. 1-5, 1991.

[10] P. A. Hageman and D. J. Blanke, "Comparison of gait of young women and elderly women," Physical therapy, vol. 66, no. 9, pp. 1382-1387, 1986.

[11] K. Supakkul, "Using positional heel-marker data to more accurately calculate stride length for treadmill walking: a step length approach," arXiv preprint arXiv:1710.09030, 2017.

[12] J. Falconer and P. W. Hayes, "A simple method to measure gait for use in arthritis clinical research," Arthritis \& Rheumatism: Official Journal of the American College of Rheumatology, vol. 4, no. 1, pp. 52-57, 1991.

[13] F. Wang, E. Stone, M. Skubic, J. M. Keller, C. Abbott, and M. Rantz, "Toward a passive low-cost in-home gait assessment sys-tem for older adults," IEEE journal of Biomedical and Health Informatics, vol. 17, no. 2, pp. 346-355, 2013.

[14] Q. Zhao, B. Zhang, J. Wang, W. Feng, W. Jia, and M. Sun, "Im-proved method of step length estimation based on inverted pendu-lum model," International journal of distributed sensor networks, vol. 13, no. 4, p. $1550147717702914,2017$.

[15] T. Liu, Y. Inoue, and K. Shibata, "A wearable ground reaction force sensor system and its application to the measurement of extrinsic gait variability," Sensors, vol. 10, no. 11, pp. 10240- 10255, 2010.

[16] G. Pulignano et al., "Incremental value of gait speed in predicting prognosis of older adults with heart failure: insights from the IM-AGE-HF study," JACC: Heart Failure, vol. 4, no. 4, pp. 289- 298, 2016.

[17] S. S. Kon et al., "Gait speed and readmission following hospitalisation for acute exacerbations of COPD: a prospective study," Thor-ax, vol. 70, no. 12, pp. 1131-1137, 2015.

[18] B. W. Schulz, J. A. Ashton-Miller, and N. B. Alexander, "The effects of age and step length on joint kinematics and kinetics of large out-and-back steps," Clinical Biomechanics, vol. 23, no. 5, pp. 609-618, 2008.

[19] D. Solanki and U. Lahiri, "Design of instrumented shoes for gait characterization: A usability study with healthy and post-stroke hemiplegic individuals," Frontiers in neuroscience, vol. 12, p. 459, 2018.

[20] E. Petersen, A. Zech, and D. Hamacher, "Walking barefoot vs. with minimalist footwear-influence on gait in younger and older adults," BMC geriatrics, vol. 20, no. 1, pp. 1-6, 2020 . 